\title{
Clinical significance of miR-155 expression in breast cancer and effects of miR-155 ASO on cell viability and apoptosis
}

\author{
SHU-RONG ZHENG ${ }^{1}$, GUI-LONG GUO ${ }^{1}$, WEI ZHANG ${ }^{1}$, GUAN-LI HUANG ${ }^{1}$, XIAO-QU HU ${ }^{1}$, \\ JIN ZHU $^{2}$, QI-DI HUANG ${ }^{1}$, JIE YOU ${ }^{1}$ and XIAO-HUA ZHANG ${ }^{1}$ \\ ${ }^{1}$ Department of Oncology, The First Affiliated Hospital of Wenzhou Medical College, \\ Zhejiang 325000; ${ }^{2}$ Department of Breast Surgery, Jiaxing Maternity and \\ Child Health Care Hospital, Zhejiang 314000, P.R. China
}

Received October 19, 2011; Accepted December 5, 2011

DOI: $10.3892 /$ or.2012.1634

\begin{abstract}
Accumulating evidence shows that mircroRNAs (miRNAs) play a vital role in tumorigenesis. miR-155 is one of the most multifunctional miRNAs whose overexpression has been found to be associated with different types of cancer including breast cancer. To further determine the potential involvement of miR-155 in breast cancer, we evaluated the expression levels of miR-155 by real-time PCR and correlated the results with clinicopathological features. Matched nontumor and tumor tissues of 42 infiltrating ductal carcinomas and 3 infiltrating lobular carcinomas were analyzed for miR-155 expression by real-time PCR. Further, we used an antisense technique to inhibit miR-155 expression in vitro. WST-8 test was performed to evaluate cell viability and apoptosis assay was used to investigate the effect of the miR-155 antisense oligonucleotide (miR-155 ASO) on HS578T cell death. The expression levels of miR-155 were significantly higher in tumor tissues than the levels in matched non-tumor tissues $(\mathrm{P}<0.001)$. Up-regulated miR-155 expression was associated with lymph node positivity $(\mathrm{P}=0.034)$, higher proliferation index $(\mathrm{Ki}-67>10 \%)(\mathrm{P}=0.019)$ and advanced breast cancer TNM clinical stage $(\mathrm{P}=0.002)$. Interestingly, we next found that miR-155 expression levels had close relations with ER status $(\mathrm{P}=0.041)$ and $\mathrm{PR}$ status $(\mathrm{P}=0.029)$. Transfection efficiency detected by flow cytometry was higher than $70 \%$, the WST-8 test showed that viability of HS578T cells was greatly reduced after transfection with miR-155 ASO compared with the scramble (SCR) group or the liposome group. The Annexin V-FITC/PI assay also indicated that transfection with miR-155 ASO promoted apoptosis.
\end{abstract}

Correspondence to: Dr Gui-Long Guo, Department of Oncology, The First Affiliated Hospital of Wenzhou Medical College, No.2, Fu Xue Road, Wenzhou, Zhejiang 325000, P.R. China

E-mail: guoguilong@sina.com

Key words: breast neoplasms, microRNAs, microRNA-155, clinicopathological features, antisense oligonucleotide, viability, apoptosis

\section{Introduction}

miRNAs are small [20-24 nucleotides (nt)] non-coding RNA gene products that have become known as important regulators of various cellular processes by post-transcriptionally modulating gene expression $(1,2)$. Currently, more than several hundred unique mature human miRNAs are known (3). miRNAs have been reported to be involved in tumorigenesis acting as oncogenes (4-6) or suppressors $(7,8)$. Several other reports have described altered expression of miRNAs in cancer tissues compared to normal tissues, suggesting that these miRNAs could potentially represent novel clinical and prognostic markers (9-12). In particular, miR-155, which is considerd as an oncomiR, has been found to be up-regulated in B cell lymphoma and carcinoma of head and neck, breast, lung, pancreas, kidney and colon (9,13-17).

To date, several studies have demonstrated the association of elevated miR-155 with late stage and poor overall survival in several types of malignancy $(9,18)$. It is well known that the prognosis of breast cancer patients is closely related with the clinicopathological features including tumor size, histological grade and the presence of lymph node or distant metastases $(19,20)$. These clinicopathological features alone or in combination, enable the identification of individuals who are at increased risk of dying of breast cancer and also who may benefit from aggressive treatment (21). However, there is rare evidence indicating the correlation of miR-155 expression with clinicopathological features in human breast carcinoma, which may help us understand the role of miR-155 in this disease.

Therefore, in this study, we used the real-time RT-PCR method to detect the expression of miR-155, which we correlated with clinicopathological features in 42 infiltrating ductal carcinomas and 3 infiltrating lobular carcinomas. Additionally, in order to analyze the effect of miR-155 on cell growth and to investigate the potential mechanism of miR-155 in breast cancer, we utilized the antisense technique to inhibit miR-155 expression in vitro.

\section{Materials and methods}

Cell line and breast tumor specimens. The breast cancer cell line (HS578T) was obtained from ATCC and grown according 
Table I. Sequences of oligonucleotide and primers for the analysis of miR-155 expression.

Gene name

Sequence $\left(5^{\prime} \rightarrow 3^{\prime}\right)$

miR-155 ASO
5'FAM SCR
Stem-loop RT primer
miR-155-F
miR-155-R
U6 snRNA-F
U6 snRNA-R

ACCCCUAUCAAGAUUAGCAUUAA

CAGUACUUUUGUGUAGUACAA

CTCAACTGGTGTCGTGGGGCAATTCAGTTGAGCCCCTATC

TGCCTCCAACTGACTCCTAC

GCGAGCACAGAATAATACGAC

CTCGCTTCGGCAGCACA

AACGCTTCACGAATTTGCGT

$\mathrm{F}$, forward primer; $\mathrm{R}$, reverse primer.

to ATCC recommended culture conditions. Fresh samples from 45 cases of human breast cancer and paired normal adjacent tissues ( $>5 \mathrm{~cm}$ from cancer tissue) were obtained from the Department of Oncology, The First Affiliated Hospital of Wenzhou Medical College (Wenzhou, China) between February 2009 and June 2009. The samples used were not subjected to preoperative radiotherapy and/or chemotherapy and all patients were treated by modified radical mastectomy. Before RNA extraction, sections were stained with H\&E for histological diagnosis and tumor cell evaluation. Only those cases with a population of at least $70 \%$ tumor cells in the section were used in this study. Tissues were preserved by snap-freeze and stored at $-80^{\circ} \mathrm{C}$. The diagnosis and histological grade of each case were independently confirmed by two pathologists based on the WHO classification. The clinical stage was classified according to the American Joint Committee on Cancer (AJCC) tumor-lymph node-metastasis (TNM) classification system (22). The relationship between the clinicopathological characteristics of the patients and miR-155 expression are summarized in Table II.

Synthesis of miR-155 ASO sequences and transfection. The mature miRNA sequences are available from the miRNA registry. The sequences of miRNA ASO were designed, according to the principle of sequences complementary to the mature mRNA. The ASO and the scrambled negative control (SCR) sequences used in this study are listed in Table I. Both of them were chemically synthesized and 2'-OMe modified by Shanghai GenePharma Co., Ltd. (Shanghai, China) and stored at $-20^{\circ} \mathrm{C}$. Twenty-four hours before transfection, HS578T cells in the exponential phase of growth were seeded in 96- or 6-well plates (Costar) and allowed to grow overnight. The cells were then transfected with oligonucleotides using Lipofectamine ${ }^{\mathrm{TM}}$ 2000 reagent (Invitrogen) in Opti-MEM I for 6 h. Transfection complexes were prepared according to the manufacturer's instructions. At the end of transfection, the cells were incubated in medium containing $10 \%$ fetal calf serum (FCS). Transfection efficiency was detected by flow cytometry.

Total RNA extraction and real-time PCR for quantitative analysis of miR-155. HS578T cells were incubated in 6-well plates and transfected with $75 \mathrm{nM}$ oligonucleotides using the Lipofectamine 2000 reagent for $24 \mathrm{~h}$. The same process was followed as described above. Total RNA from the tissues or treated HS578T cells was isolated using TRIzol reagent (Invitrogen) according to the manufacturer's instructions. RNA purity and concentration were controlled by UV spectrophotometry (A260:A280>1.8).

The primers for the analysis of miR-155 expression were designed by the Shanghai Sangon Biological Engineering Technology and Services Co., Ltd. (Shanghai, China) and are summarized in Table I. Mixtures of $1 \mu \mathrm{g}$ of total RNAs together with $50 \mathrm{nM}$ reverse primer, 2 units of RNAase inhibitor (Takara Bio), 5 units of M-MLV reverse transcriptase (Takara Bio) and $0.5 \mu \mathrm{M}$ dNTP were used for each RT reaction. The reaction parameters were incubation at $25^{\circ} \mathrm{C}$ for $10 \mathrm{~min}, 42^{\circ} \mathrm{C}$ for $60 \mathrm{~min}, 52^{\circ} \mathrm{C}$ for $15 \mathrm{~min}, 70^{\circ} \mathrm{C}$ for $15 \mathrm{~min}$ and then a hold at $-20^{\circ} \mathrm{C}$. At the same time, to generate the cDNA template for the endogenous control PCR reactions, first strand cDNA was synthesized using $1 \mu \mathrm{g}$ of RNA from the same samples for stem-loop reverse transcription and oligo(dT) as the primer. The reaction parameters were incubation at $42^{\circ} \mathrm{C}$ for $30 \mathrm{~min}$, $70^{\circ} \mathrm{C}$ for $15 \mathrm{~min}$ and then a hold at $-20^{\circ} \mathrm{C}$.

The qPCR was performed on the Applied Biosystems 7500 detection system. For quantitation of miR-155, the $25 \mu \mathrm{l}$ PCR included $1 \mu \mathrm{l}$ of the RT product of miR-155, 1X SYBR-Green I Mastermix (Toyobo Co., Ltd.), $0.5 \mu \mathrm{M}$ specific forward primer of miR-155 and $0.5 \mu \mathrm{M}$ reverse primer. For the endogenous control, U6 snRNAs, $1 \mu \mathrm{l}$ of cDNAs synthesized by using oligo(dT) were used as a template. The reaction parameters were incubation at $95^{\circ} \mathrm{C}$ for $10 \mathrm{~min}$, then 40 cycles of $95^{\circ} \mathrm{C}$ for $15 \mathrm{sec}$ and $60^{\circ} \mathrm{C}$ for $1 \mathrm{~min}$. To calculate the relative concentration, miR-155 and U6 CT values for all samples were obtained. A normalized expression for each sample was obtained by dividing the $\mathrm{Ct}$ value of miR-155 by the same sample's $\mathrm{U} 6 \mathrm{Ct}$ and designated as $\Delta \mathrm{Ct}$. This value was then transformed by the formula $2^{-\Delta \mathrm{Ct}}$. Furthermore, the $(\Delta \Delta \mathrm{Ct})$ method was used in comparing miR-155 expression in each group of treated HS578T cells or matched non-tumor tissue to cancer tissues.

Cell viability and apoptosis assays. The effect of miR-155 ASO on HS578T cell viability was determined by the 2-(2-methoxy-4-nitrophenyl)-3-(4-nitrophenyl)-5-(2,4-disulfophenyl)-2H-tetrazolium, monosodium salt (WST-8) assay kit (CCK-8, Dojindo, Kumamoto, Japan). Twenty-four hours before transfection, 1 $\times 10^{4}$ HS578T cells/well were seeded in 96-well plates and allowed to grow overnight. The cells were then transfected with three different concentrations of miR-155 
ASO (25, 50 and $75 \mathrm{nM})$ and the highest concentration of SCR siRNA (75 nM) using Lipofectamine 2000 according to the manufacturer's protocol. After $24 \mathrm{~h}$, WST- 8 was added into each well for $1 \mathrm{~h}$ before the measurement according to the manufacturer's instructions. The absorbance at $450 \mathrm{~nm}$ was measured by a microplate reader. The following formula was used for calculating the inhibition rate. Inhibition rate $=$ (1-absorbance of treated cells/control cells) x 100\% (23). The apoptosis assay was performed with the Annexin V-FITC/PI Apoptosis Detection kit (Roche). HS578T cells were transfected with miR-155 ASO or SCR siRNA as previously described for $6 \mathrm{~h}$, and incubated in medium containing $10 \%$ FCS for another $24 \mathrm{~h}$ in 6-well plates. Cells were collected and double-stained with FITC-conjugated Annexin V and propidium iodide (PI). For each sample, data from approximately $1 \times 10^{4}$ cells were recorded in the list mode on logarithmic scales. Apoptosis and necrosis were analyzed by quadrant statistics on PI-negative, Annexin V-positive cells and both positive cells, respectively.

Statistical analysis. For all data, statistical analysis was performed in SPSS 17.0 for Windows (SPSS, Inc.). The miR-155 expression levels were characterized by their median and ranges from the 25th to the 75th percentile. The Wilcoxon test was used for comparing two paired groups (tumor and paired non-tumor), the Mann-Whitney $\mathrm{U}$ test for two independent groups and the Kruskall-Wallis test for three independent groups (relationship between miR-155 expression level and TNM stage of breast cancer). The one-way ANOVA test was performed to investigate the differences in the obtained results of the WST- 8 array and apoptosis analysis. All tests were twotailed and the significance level was set at $\mathrm{P}<0.05$.

\section{Results}

Expression of miR-155 in tumor tissue and matched nontumor tissue. The melting-curves of miR-155 and U6 snRNA were sharply defined curves with a narrow peak (Fig. 1A), indicating that pure, homogeneous PCR products were produced. As shown in the amplification curves (Fig. 1B), the Ct value of miR-155 in tumor tissue was lower than that of miR-155 in non-tumor tissue, which means the expression level of miR-155 in tumor samples was higher than that in the controls. The median of the relative expression of miR-155 $\left(2^{-\Delta \Delta \mathrm{Ct}}\right)$ was 0.360 (25th-75th percentile, $\left.0.328-0.420\right)$ in tumor samples, with that in non-tumor control samples set at 0.135 (25th-75th percentile, 0.003-0.199) (Fig. 1C). The difference of expression of miR-155 between the tumor and the control samples was statistically significant $(P<0.001$, Wilcoxon test).

Association between miR-155 expression level and clinicopathological parameters. The up-regulated expression of miR-155 was associated with advanced clinical TNM stage ( $\mathrm{P}=0.002$, Kruskall-Wallis test), lymph node positivity ( $\mathrm{P}=0.034$, Mann-Whitney $\mathrm{U}$ test) and high proliferation index (Ki-67 >10\%) ( $\mathrm{P}=0.019$, Mann-Whitney U test). Furthermore, the miR-155 expression levels were closely related to ER and PR status $(\mathrm{P}=0.041$ and 0.029 , respectively, by the MannWhitney U test). However, no significant relationship was found between the expression of miR-155 and the menstrual
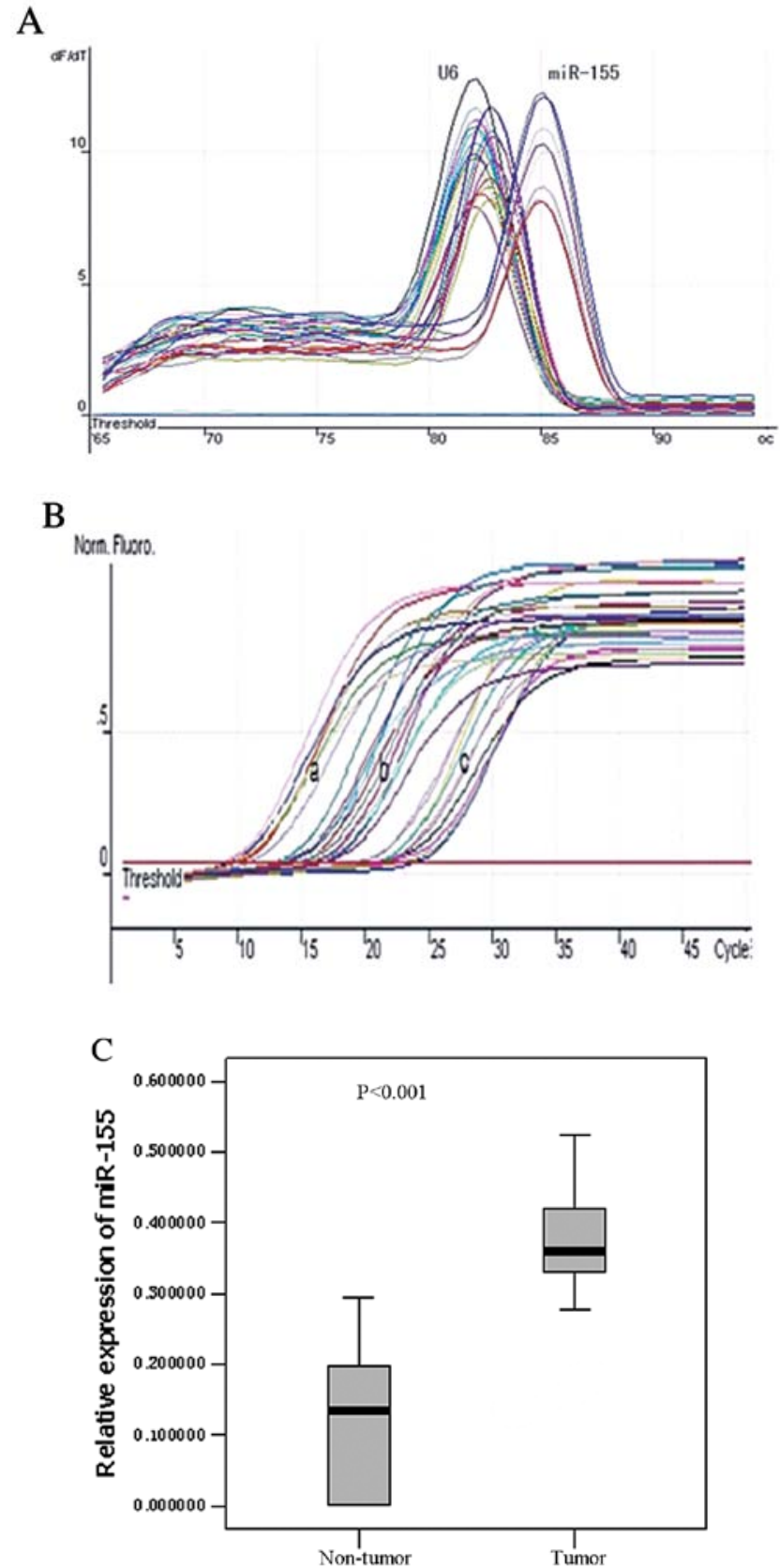

Figure 1. miR-155 expression in breast cancer. (A) The melting-curves of miR-155 and U6 snRNA are presented as a single, sharply-defined melting curve with a narrow peak, indicating that pure, homogeneous PCR products were produced. (B) Representative amplification curves show that the $\mathrm{Ct}$ values of miR-155 in the tumor tissue are lower than in the non-tumor tissue, indicating the miR-155 expression is higher in tumor tissue than in non-tumor tissue. Reactions were performed in triplicate. (A) U6 snRNA of tumor and matched non-tumor; (B) miR-155 of tumor; (C) miR-155 of matched nontumor. (C) Differences in the expression levels of miR-155 between the tumor tissue and matched non-tumor tissue (box-plot diagrams with median, 1st quartile, 3rd quartile and non-outlier range).

status $(\mathrm{P}=0.640)$, size of primary tumor $(\mathrm{P}=0.530)$, Her-2 status $(\mathrm{P}=0.647)$ and pathologic type $(\mathrm{P}=0.559)$ using the Mann-Whitney U test (Table II).

miR-155 ASO down-regulation of miRNA expression. Eight hours after transfection with $75 \mathrm{nM}$ 5'FAM SCR, the transfection efficiency was detected by flow cytometry (Fig. 2A). To validate whether miR-155 ASO decreased miR-155 levels 
Table II. Relationship between the miR-155 expression level and clinicopathological parameters of breast cancer.

\begin{tabular}{|c|c|c|c|}
\hline Variable & $\mathrm{N}$ & $\begin{array}{c}\text { Relative expression } \\
\text { of } \mathrm{miR}-155^{\mathrm{a}}\end{array}$ & P-value ${ }^{b}$ \\
\hline Menstrual status & & & 0.640 \\
\hline Premenopausal & 20 & $0.365(0.333-0.421)$ & \\
\hline Post-menopausal & 25 & $0.355(0.308-0.403)$ & \\
\hline Tumor size $(\mathrm{cm})^{\mathrm{c}}$ & & & 0.530 \\
\hline$\leq 2$ & 19 & $0.356(0.316-0.401)$ & \\
\hline$>2$ & 26 & $0.361(0.334-0.420)$ & \\
\hline TNM stage & & & 0.002 \\
\hline $\mathrm{I}$ & 8 & $0.316(0.286-0.350)$ & \\
\hline II & 24 & $0.358(0.332-0.416)$ & \\
\hline III & 13 & $0.417(0.367-0.446)$ & \\
\hline ER status & & & 0.041 \\
\hline Positive & 30 & $0.367(0.349-0.424)$ & \\
\hline Negative & 15 & $0.318(0.299-0.401)$ & \\
\hline PR status & & & 0.029 \\
\hline Positive & 26 & $0.398(0.354-0.423)$ & \\
\hline Negative & 19 & $0.335(0.313-0.399)$ & \\
\hline Her-2 status & & & 0.647 \\
\hline Positive & 14 & $0.398(0.313-0.420)$ & \\
\hline Negative & 31 & $0.359(0.337-0.419)$ & \\
\hline Lymph node status & & & 0.034 \\
\hline Metastasis & 20 & $0.383(0.355-0.437)$ & \\
\hline No metastasis & 25 & $0.355(0.314-0.399)$ & \\
\hline Proliferation & & & \\
\hline index $(\mathrm{Ki}-67)(\%)$ & & & 0.019 \\
\hline$\leq 10$ & 21 & $0.353(0.364-0.398)$ & \\
\hline$>10$ & 24 & $0.387(0.355-0.437)$ & \\
\hline Pathologic type & & & 0.559 \\
\hline Ductal & 42 & $0.360(0.331-0.420)$ & \\
\hline Lobular & 3 & $0.330(0.277-0.442)$ & \\
\hline
\end{tabular}

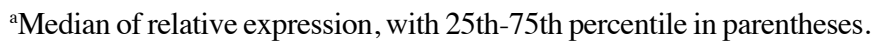
${ }^{\mathrm{b}} \mathrm{P}<0.05$ was considered significant (Mann-Whitney U test between 2 groups and Kruskall-Wallis test for 3 groups). ${ }^{\mathrm{c}}$ Maximal tumor diameter.

in treated HS578T cells, miR-155 and U6 snRNA expression was determined by real-time RT-PCR. The fold change for the miR-155 expression level was calculated using the $2^{-\Delta \Delta C t}$ method. As shown in Fig. 2B, the $2^{-\Delta \Delta \mathrm{Ct}}$ value of HS578T cells treated with anti-miR-155 was 0.052 , which showed that the level of miR-155 in HS578T cells was down-regulated by miR-155 ASO.

miR-155 ASO inhibition of HS578T cells viability. In this study, we determined the influence of miR-155 ASO on cell viability by WST- 8 assay. Optical densities at $450 \mathrm{~nm}$ were obtained for 4 groups: $0.984 \pm 0.090$ for control group, $0.949 \pm 0.061$ for liposomes group, $0.923 \pm 0.096$ for SCR group and $0.905 \pm 0.024$, $0.765 \pm 0.095$ and $0.551 \pm 0.037$, respectively for 25,50 and $75 \mathrm{nM}$ concentrations of miR-155 ASO (Fig. 3). These results demonstrated that the optical density and therefore the cell viability
A
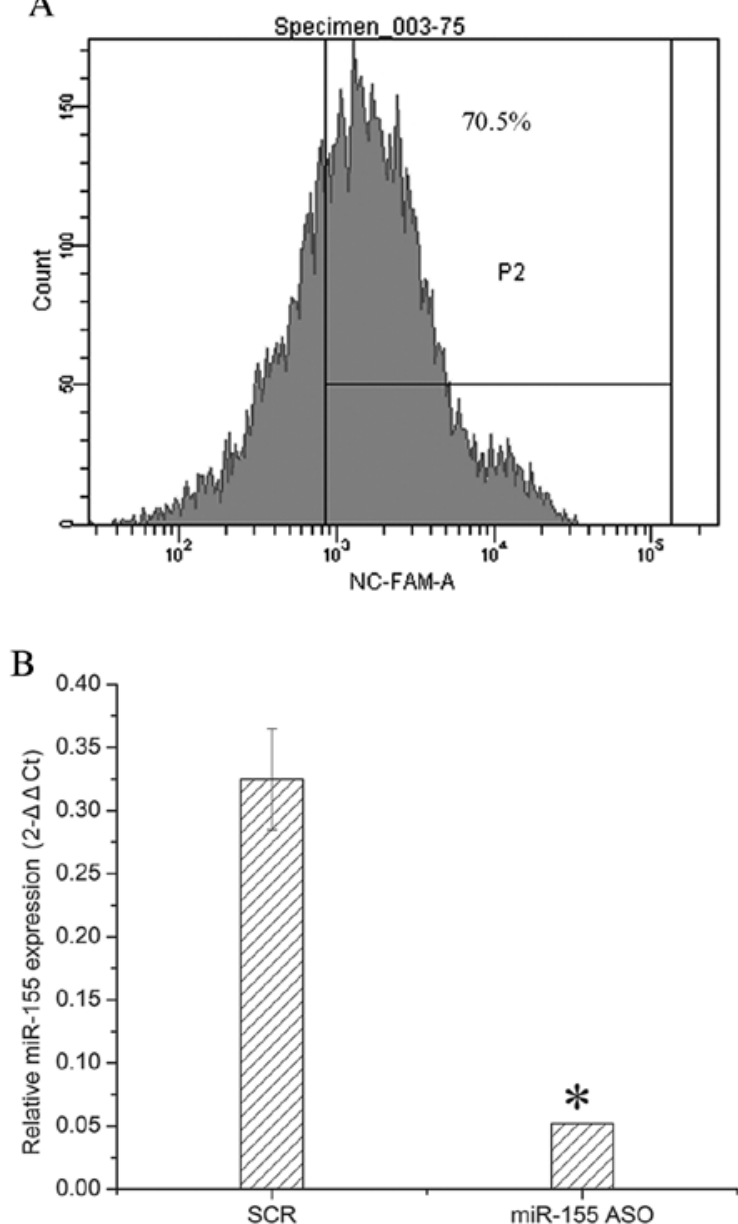

Figure 2. Detection of transfection efficiency and miR-155 expression ratio relative to U6 snRNA in HS578T cells. (A) Eight hours after transfection with $75 \mathrm{nM}$ 5'FAM SCR, the transfection efficiency was detected by flow cytometry. The $\mathrm{P} 2$ region stands for the number of cells that were successfully transfected with 5'FAM SCR by Lipofectamine 2000. (B) HS578T cells were transfected with $75 \mathrm{nM}$ miR-155 ASO or SCR. Total RNA from treated cells was extracted by TRIzol and quantified by ultraviolet spectrophotometry. miR-155 and U6 snRNA expression were determined by quantitative real-time PCR, according to the manufacturer's instructions. Results showed that miR-155 ASO down-regulated miR-155 levels in HS578T cells. ${ }^{*} \mathrm{P}<0.01$, compared with the SCR group.

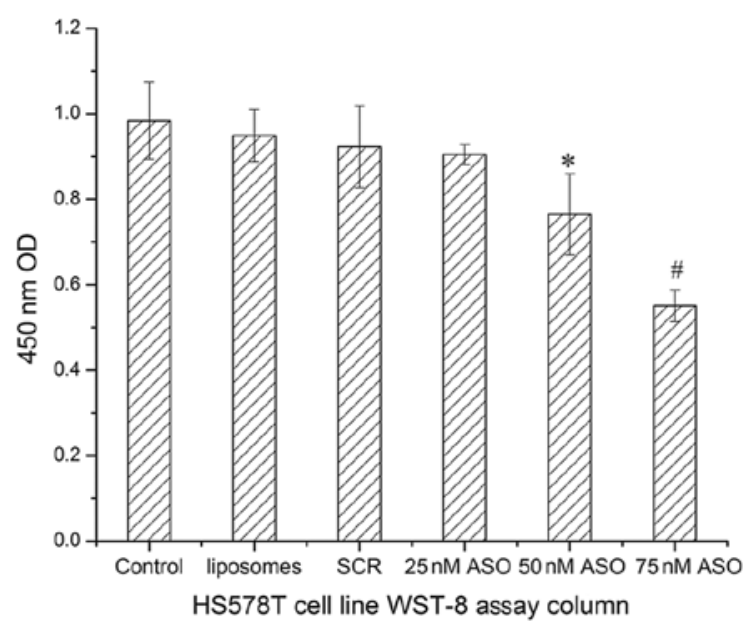

Figure 3. WST-8 assay on control, liposomes, SCR and test groups. $\mathrm{P}=0.000$ compared to control group; ${ }^{\#} \mathrm{P}=0.000$ compared to control group. 
A

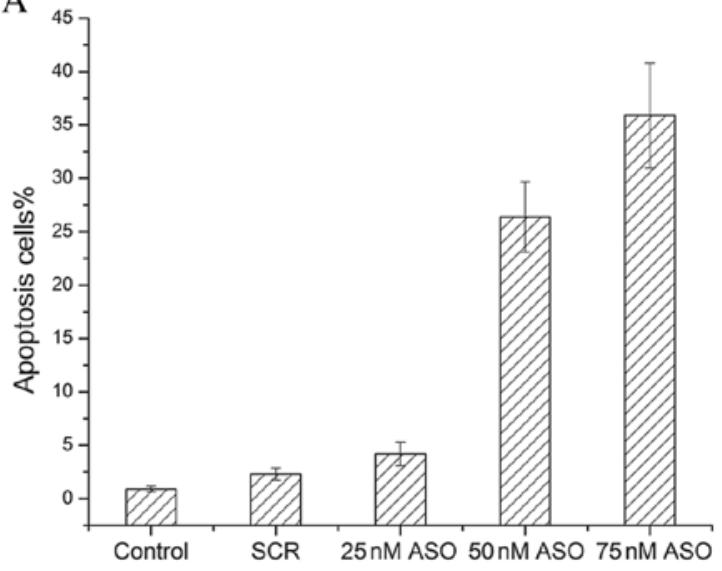

C

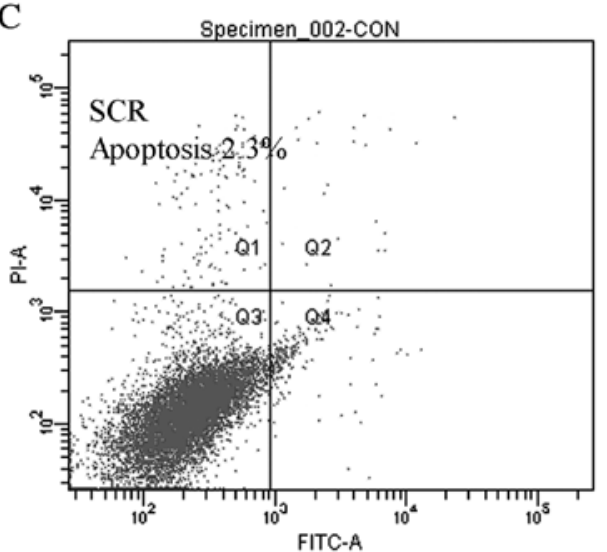

E

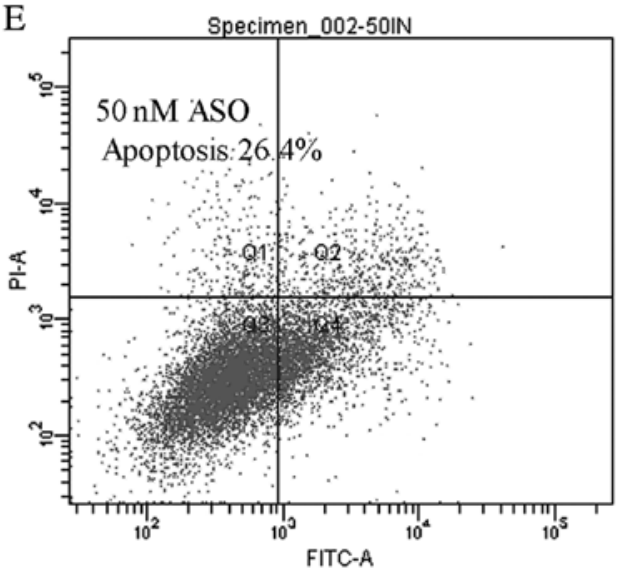

B

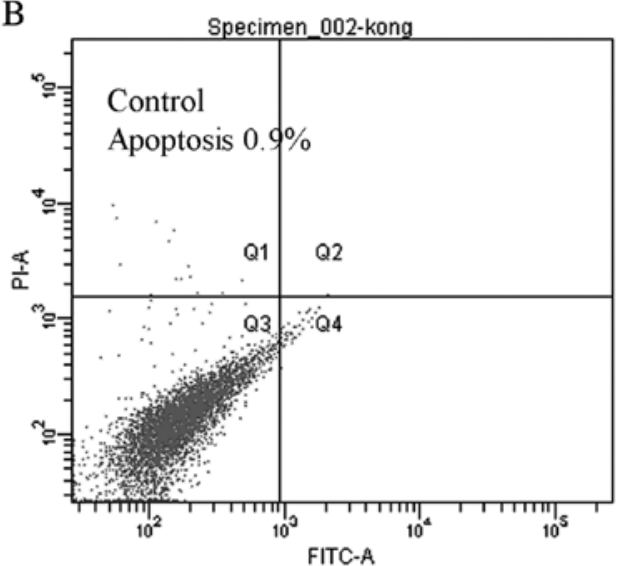

D

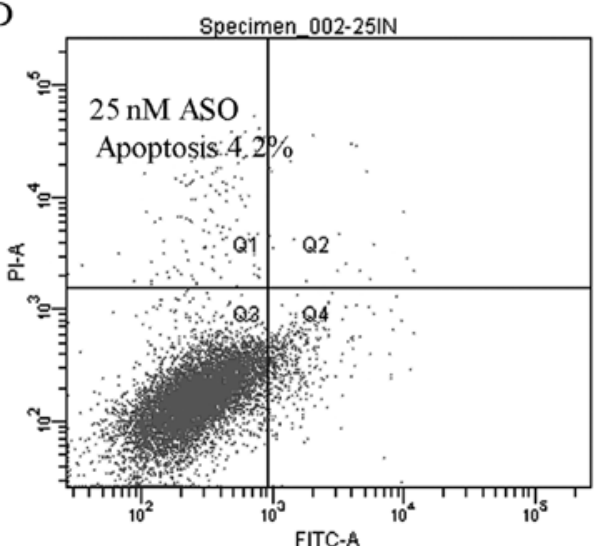

F

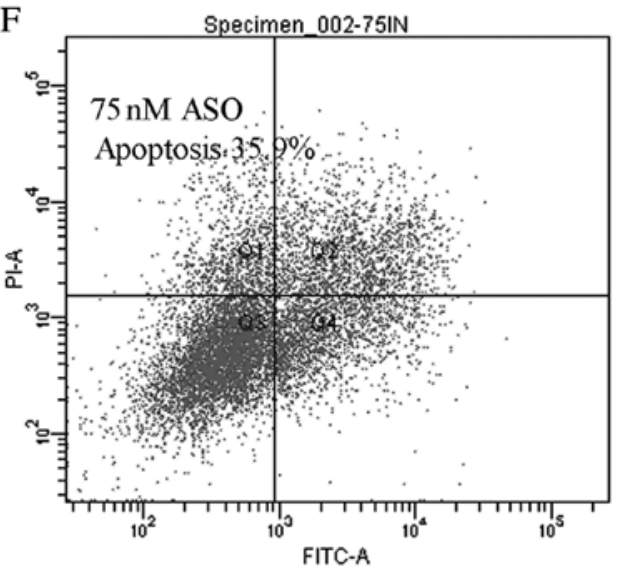

Figure 4. Induction of HS578T cell apoptosis by miR-155 ASO. (A) HS578T cells were transfected with miR-155 ASO or SCR for 6 h and incubated in medium containing $10 \%$ FCS for another $24 \mathrm{~h}$. Apoptosis of HS578T cells were double-stained with Annexin V and PI, and detected by flow cytometry. miR-155 ASO 50 and $75 \mathrm{nM}$ could efficiently induced apoptosis ( $\mathrm{P}<0.01$ as compared with SCR). (B-F) The images of apoptosis were analyzed by double staining with Annexin V and PI.

was similar in the control, SCR, $25 \mathrm{nM}$ miR-155 ASO and liposome groups ( $\mathrm{P}=0.237$, one-way ANOVA test). However, there were significant differences between the optical density in the $50 \mathrm{nM}$ miR-155 ASO and $75 \mathrm{nM}$ miR-155 ASO group and the control group ( $\mathrm{P}=0.000$ for both by one-way ANOVA). The optical density and cell viability gradually decreased with the increase of miR-155 ASO concentration (0.905 \pm 0.024 , $0.765 \pm 0.095$ and $0.551 \pm 0.037$, respectively). At $75 \mathrm{nM}$ concentration of miR-155 ASO, the optical density and cell viability were nearly half of these parameters in the control group. These data indicate that a higher concentration of miR-155 ASO had a higher toxicity effect on HS578T cells and could decrease the cell viability and proliferation.

miR-155 ASO promotion of HS578T cell apoptosis. To explore the effects of miR-155 ASO on cell apoptosis, miR-155 ASO treatment was investigated in HS578T cells. Apoptotic HS578T cells were detected by double staining with Annexin V and PI. The results demonstrated that miR-155 ASO could induce cell apoptosis. Along with the increase of concentration of miR-155 ASO, the apoptosis rate of HS578T cells gradually increased. The double-stained images are shown in Fig. 4. 


\section{Discussion}

As previous research has indicated, miR-155 acts as a multifunctional miRNA in many pathophysiological process, such as immunology $(24,28)$, inflammation $(25,26)$, hematopoiesis (27), angiocardiopathy (28) and carcinogenesis. Interestingly, miR-155 may act as a bridge between inflammation and malignancy, which may provide new insight in carcinogenesis (29-31). miR-155 is one of the most prominent miRNAs involved in tumor development and progression. Diverse studies have shown that miR-155 is overexpressed in various tumor types. Not surprisingly, it was found to be significantly up-regulated in breast cancer in both our and previous other studies $(15,29,32)$. However, there is rare evidence focused on the relationship between miR-155 expression and clinicopathological features in breast cancer.

In the first stage of our study, we detected the expression of miR-155 in all 45 carcinomas and paired non-tumor tissues by real-time RT-PCR. The amplification and melting curve, as shown in Fig. 1, indicate the method is specific and sensitive enough for detection of miR-155. Furthermore, to reduce the error caused by gene expression differences between different individuals, we used matched non-tumor tissue as control and used $2^{-\Delta \Delta \mathrm{Ct}}$ to represent the level of miR-155 expression in tumors relative to matched non-tumor samples. As expected, our results confirmed that miR-155 expression was significantly up-regulated in breast cancer compared with the matched non-tumor tissue.

Our study next focused on the potential correlation between the expression level of miR-155 and various breast cancer clinicopathological characteristics. The data showed that high levels of miR-155 appear to be significantly correlated with advanced clinical stage, lymph node metastases, and higher proliferation index (Ki-67 >10\%). All of them are well known as poor prognostic factors of breast cancer patients (33-35). Interestingly, we next found that patients with ER-positive or PR-positive tumors have higher miR-155 expression levels than those that are ER-negative or PR-negative. This is in part consistent with the findings of Zhu et al (36) and Lu and Tsourkas (37), whose experimental material was either serum or cells but not tissues. However, it should be noted that both ER and PR are protective factors of patients with breast cancer (38-40). Therefore, the representation of the oncomiR-like miR-155 is contradictory to the protective function of ER and $\mathrm{PR}$. And we may hypothesize that it is miR-155 that results in the aggressive behavior in carcinomas that ER- or PR-positive. These results indicate that, as an independent risk factor, miR-155 could serve as a prognostic marker for survival of breast cancer patients.

The precise molecular mechanisms behind the altered expression of miR-155 in breast cancer remain poorly understood. To our knowledge, this is the first report to describe the significance of miR-155 to the clinical stage, lymph node metastasis, hormone receptor status of breast cancer patients. It was recently reported that miR-155 mediates lymphoblastoid cell lines (LCLs); suppression of this miRNA, which is highly expressed in LCLs, was associated with decreased cell proliferation and increased apoptosis (41). In solid tumors, such as breast cancer and lung cancer, similar findings of the function for miR-155 were obtained $(29,42)$. Furthermore, Kong et al
(15) discovered that miR-155 is a critical therapeutic target and is closely related with chemosensitivity in breast cancer. Two recent studies reported two additional direct miR-155 targets, FOXO3a (15) and suppressor of cytokine signaling 1 (29), respectively, both of which function as protective factors in breast cancer patients, demonstrating that this miRNA acts as an oncomiR in breast cancer.

Therefore, in this study, we utilized miR-155 ASO for repression of HS578T cell growth and proliferation. Transfection of HS578T cells with either miR-155 ASO or SCR were performed successfully with at least $70 \%$ efficiency. The results of real-time PCR suggested that the synthesized miR-155 ASO could effectively down-regulate this miRNA expression. The WST- 8 assay was performed and the results indicated that although 25 and $50 \mathrm{nM}$ of miR-155 ASO have a toxic effect on HS578T cells, $75 \mathrm{nM}$ of miR-155 ASO could strongly repress tumor cell proliferation. Moreover, apoptosis analysis demonstrated that the apoptosis rates of the group of 50 and $75 \mathrm{nM}$ of miR-155 ASO were significantly higher than the other three groups.

In conclusion, we demonstrated that overexpression of miR-155, one of the most significantly altered miRNAs in breast cancer, is related to clinical stage, lymph node metastasis, higher Ki-67 and hormone receptor status of breast cancer patients. Although the precise molecular mechanism of the ectopic expression of miR-155 in breast cancer requires further clarification, our data suggest that miR-155 may be a promising candidate as a molecular biomarker and a potential therapeutic target for breast cancer intervention.

\section{Acknowledgements}

This study was supported by grants from the Wenzhou Science and Technology Bureau (Y20080081, Y20100008).

\section{References}

1. Ambros V: MicroRNA pathways in flies and worms: growth, death, fat, stress, and timing. Cell 113: 673-676, 2003.

2. Bartel DP: MicroRNAs: target recognition and regulatory functions. Cell 136: 215-233, 2009.

3. Griffiths-Jones S, Grocock RJ, van Dongen S, Bateman A and Enright AJ: miRBase: microRNA sequences, targets and gene nomenclature. Nucleic Acids Res 34: D140-D144, 2006.

4. He L, Thomson JM, Hemann MT, et al: A microRNA polycistron as a potential human oncogene. Nature 435: 828-833, 2005.

5. Tam W and Dahlberg JE: miR-155/BIC as an oncogenic microRNA. Genes Chromosomes Cancer 45: 211-212, 2006.

6. Papagiannakopoulos T, Shapiro A and Kosik KS: MicroRNA-21 targets a network of key tumor-suppressive pathways in glioblastoma cells. Cancer Res 68: 8164-8172, 2008.

7. Hammond SM: MicroRNAs as tumor suppressors. Nat Genet 39: 582-583, 2007.

8. Tazawa H, Tsuchiya N, Izumiya $M$ and Nakagama H: Tumorsuppressive miR-34a induces senescence-like growth arrest through modulation of the E2F pathway in human colon cancer cells. Proc Natl Acad Sci USA 104: 15472-15477, 2007.

9. Yanaihara N, Caplen N, Bowman E, et al: Unique microRNA molecular profiles in lung cancer diagnosis and prognosis. Cancer Cell 9: 189-198, 2006.

10. Mattie MD, Benz CC, Bowers J, et al: Optimized high-throughput microRNA expression profiling provides novel biomarker assessment of clinical prostate and breast cancer biopsies. Mol Cancer 5: 24, 2006.

11. Chen Y and Stallings RL: Differential patterns of microRNA expression in neuroblastoma are correlated with prognosis, differentiation, and apoptosis. Cancer Res 67: 976-983, 2007. 
12. Ru Y, Dancik GM and Theodorescu D: Biomarkers for prognosis and treatment selection in advanced bladder cancer patients. Curr Opin Urol 21: 420-427, 2011.

13. Eis PS, Tam W, Sun L, et al: Accumulation of miR-155 and BIC RNA in human B cell lymphomas. Proc Natl Acad Sci USA 102: 3627-3632, 2005.

14. Wald AI, Hoskins EE, Wells SI, Ferris RL and Khan SA: Alteration of microRNA profiles in squamous cell carcinoma of the head and neck cell lines by human papillomavirus. Head Neck 33: 504-512, 2011.

15. Kong W, He L, Coppola M, Guo J, Esposito NN, Coppola D and Cheng JQ: MicroRNA-155 regulates cell survival, growth, and chemosensitivity by targeting FOXO3a in breast cancer. J Biol Chem 285: 17869-17879, 2010.

16. Volinia S, Calin GA, Liu CG, et al: A microRNA expression signature of human solid tumors defines cancer gene targets. Proc Natl Acad Sci USA 103: 2257-2261, 2006.

17. Juan D, Alexe G, Antes T, et al: Identification of a microRNA panel for clear-cell kidney cancer. Urology 75: 835-841, 2010.

18. Du ZM, Hu LF, Wang HY, Yan LX, Zeng YX, Shao JY and Ernberg I: Upregulation of MiR-155 in nasopharyngeal carcinoma is partly driven by LMP1 and LMP2A and downregulates a negative prognostic marker JMJD1A. PLoS One 6: e19137, 2011.

19. Bundred NJ: Prognostic and predictive factors in breast cancer. Cancer Treat Rev 27: 137-142, 2001

20. Devi KR, Kuruvila S and Musa MM: Pathological prognostic factors in breast carcinoma. Saudi Med J 21: 372-375, 2000.

21. Wiseman SM, Makretsov N, Nielsen TO, et al: Coexpression of the type 1 growth factor receptor family members HER-1, HER-2, and HER-3 has a synergistic negative prognostic effect on breast carcinoma survival. Cancer 103: 1770-1777, 2005.

22. Singletary SE, Allred C, Ashley P, et al: Staging system for breast cancer: revisions for the 6th edition of the AJCC Cancer Staging Manual. Surg Clin North Am 83: 803-819, 2003.

23. Zhang RG, Zhang RP, Wang XW and Xie H: Effects of cisplatin on telomerase activity and telomere length in BEL-7404 humanhepatoma cells. Cell Res 12: 55-62, 2002.

24. O'Connell RM, Taganov KD and Boldin MP: MicroRNA-155 is induced during the macrophage inflammatory response. Proc Natl Acad Sci USA 104: 1604-1609, 2007.

25. O'Connell RM, Kahn D, Gibson WS, et al: MicroRNA-155 promotes autoimmune inflammation by enhancing inflammatory T cell development. Immunity 33: 607-619, 2010.

26. Bhattacharyya S, Balakathiresan NS, Dalgard C, et al: Elevated miR-155 promotes inflammation in cystic fibrosis by driving hyperexpression of interleukin-8. J Biol Chem 286: 11604-11615, 2011.

27. O'Connell RM, Rao DS, Chaudhuri AA, et al: Sustained expression of microRNA-155 in hematopoietic stem cells causes a myeloproliferative disorder. J Exp Med 205: 585-594, 2008.
28. Urbich C, Kuehbacher A and Dimmeler S: Role of microRNAs in vascular diseases, inflammation, and angiogenesis. Cardiovasc Res 79: 581-588, 2008.

29. Jiang S, Zhang HW, Lu MH, et al: MicroRNA-155 functions as an OncomiR in breast cancer by targeting the suppressor of cytokine signaling 1 gene. Cancer Res 70: 3119-3127, 2010.

30. Pedersen IM, Otero D, Kao E, Miletic AV, et al: Onco-miR-155 targets SHIP1 to promote TNFalpha-dependent growth of B cell lymphomas. EMBO Mol Med 1: 288-295, 2009.

31. Tili E, Croce CM and Michaille JJ: miR-155: on the crosstalk between inflammation and cancer. Int Rev Immunol 28: 264-284, 2009.

32. Iorio MV, Ferracin M, Liu CG, et al: MicroRNA gene expression deregulation in human breast cancer. Cancer Res 65: 7065-7070, 2005.

33. Wrba F, Reiner A, Markis-Ritzinger E, Holzner JH, Reiner G and Spona J: Prognostic significance of immunohistochemical parameters in breast carcinomas. Pathol Res Pract 183: 277-283, 1988.

34. Carter CL, Allen C and Henson DE: Relation of tumor size, lymph node status, and survival in 24,740 breast cancer cases. Cancer 63: 181-187, 1989.

35. De Azambuja E, Cardoso F, de Castro G Jr, et al: Ki-67 as prognostic marker in early breast cancer: a meta-analysis of published studies involving 12,155 patients. Br J Cancer 96: 1504-1513, 2007.

36. Zhu W, Qin W, Atasoy U and Sauter ER: Circulating microRNAs in breast cancer and healthy subjects. BMC Res Notes 2: 89, 2009.

37. Lu J and Tsourkas A: Imaging individual microRNAs in single mammalian cells in situ. Nucleic Acids Res 37: e100, 2009.

38. Lee YT and Markland FS: Steroid receptors study in breast carcinoma. Med Pediatr Oncol 5: 153-166, 1978

39. Paone JF, Abeloff MD, Ettinger DS, Arnold EA and Baker RR: The correlation of estrogen and progesterone receptor levels with response to chemotherapy for advanced carcinoma of the breast. Surg Gynecol Obstet 152: 70-74, 1981.

40. Clark GM and McGuire WL: Prognostic factors in primary breast cancer. Breast Cancer Res Treat 3 (Suppl): S69-S72, 1983.

41. Linnstaedt SD, Gottwein E, Skalsky RL, Luftig MA and Cullen BR: Virally induced cellular microRNA miR-155 plays a key role in B-cell immortalization by Epstein-Barr virus. J Virol 84: 11670-11678, 2010

42. Qin A, Zhou Y, Sheng M, Fei G, Ren T and Xu L: Effects of microRNA-155 on the growth of human lung cancer cell line 95D in vitro. Zhongguo Fei Ai Za Zhi 14: 575-580, 2011. 